\title{
Evaluación de los costos de construcción de sistemas estructurales para viviendas de baja altura y de interés social
}

\author{
Construction Costs Assessment of Structural Systems for \\ Low-Rise and Social Welfare Housing
}

\author{
Carrillo Julián \\ Universidad Militar Nueva Granada, Bogotá, Colombia \\ Departamento de Ingeniería Civil \\ Correo:wjcarrillo@gmail.com \\ Echeverri Fabián \\ Universidad Militar Nueva Granada, Bogotá, Colombia \\ Departamento de Ingeniería Civil \\ Correo:fabyanecheverry@gmail.com
}

\author{
Aperador William \\ Universidad Militar Nueva Granada, Bogotá, Colombia \\ Facultad de Ingeniería \\ Correo:wiliam.aperador@unimilitar.edu.co
}

Información del artículo: recibido: febrero de 2014, aceptado: febrero de 2015

\section{Resumen}

Se realizó un análisis comparativo de los costos asociados a la construcción de vivienda de baja altura, bajo costo y de interés social. El estudio incluyó tres de los sistemas estructurales más utilizados para la construcción de vivienda de baja altura en Latinoamérica, tales como el sistema tradicional de muros de mampostería confinada, muros de concreto reforzados convencionalmente con mallas electrosoldadas y muros de concreto reforzados con fibras de acero. La comparación de los costos se llevó a cabo mediante el análisis de presupuestos, los cuales se realizaron con base en las cantidades de obra, los precios unitarios y los ítems particulares de cada sistema estructural. En el estudio se determinó que, desde el punto de vista económico, los sistemas de muros de concreto reforzados con mallas electrosoldadas o con fibras de acero son más favorables que los sistemas de mampostería confinada. Adicionalmente, la comparación integral de los tres sistemas estructurales demuestra que el sistema industrializado de muros de concreto reforzado con fibras de acero permite obtener mayores beneficios de limpieza y sostenibilidad, mayor velocidad de construcción, menor costo y un panorama más atractivo para los constructores que invierten en este tipo de proyectos.

\section{Descriptores:}

- vivienda

- baja altura

- bajo costo

- interés social

- muros de concreto

- muros de mampostería confinada

- malla electrosoldada

- fibras de acero

- sistema industrializado

- costos 


\begin{abstract}
A comparative analysis of the costs related to the construction of low-rise, low-cost and social welfare housing was carried out. The study included three of the most commonly used structural systems for low-rise housing in Latin America, such as the traditional system of confined masonry walls, concrete walls conventionally reinforced with welded-wire meshes and concrete walls reinforced with steel fiber. The cost comparison was carried out by budgets analysis, which were performed based on construction quantities, unit prices and particular items for each structural system. It was found in the study that, from an economic point of view, the systems of concrete walls reinforced with welded-wire meshes or steel fibers are more advantageous than confined masonry systems. In addition, the integral comparison of the three structural systems demonstrates that the industrialized system of steel fiber reinforced concrete walls allows obtaining greater advantages of cleaning and sustainability, faster construction, lower cost and a more attractive scenario for builders investing in such projects.
\end{abstract}

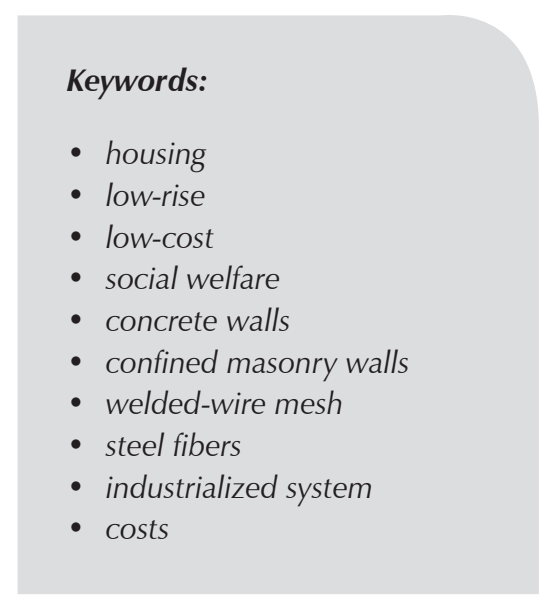

\section{Introducción}

Durante los últimos años en Latinoamérica se ha observado un incremento importante en la construcción de viviendas de interés social (VIS), localizadas en todo tipo de climas y en todas las zonas de amenaza sísmica. Según el Departamento Administrativo Nacional de Estadística de Colombia, DANE (2013), entre julio de 2010 y octubre de 2013, la participación promedio de las unidades licenciadas de VIS sobre el total de unidades licenciadas de vivienda fue de $43.5 \%$. En el mes de octubre de 2013, la participación fue de $51.5 \%$, la cual es superior a la participación del mismo mes de 2012 (35.1\%). Respecto a las unidades licenciadas, la vivienda VIS creció $135.5 \%$ respecto al mismo mes del año inmediatamente anterior.

La infraestructura de viviendas tipo VIS requiere la construcción de varias unidades habitacionales. Estas viviendas deben prestar un buen nivel de servicio, y deben cumplir con los requisitos mínimos de calidad y de seguridad estipulados por los Reglamentos de Construcción Sismo-Resistente; por ejemplo, en Colombia, las viviendas deben cumplir con los requisitos estipulados en la NSR-10 (AIS, 2010), el Decreto 2060 de 2004 (Minvivienda, 2004), el Decreto 619 de 2000 (SPD, 2000) y la Ley 1450 de 2011 (Minhacienda y DNP, 2011). Adicionalmente, para cumplir con las demandas crecientes y con los costos reducidos, estas viviendas deben construirse en el menor tiempo y con el menor costo posible. Por consiguiente, para definir el sistema constructivo que permita realizar la construcción de VIS más eficiente y sin descuidar los estándares de calidad y de servicio, es necesario analizar los costos totales asociados a los sistemas estructurales más utilizados para este tipo de viviendas.
En este artículo se presentan los resultados del análisis comparativo de los costos asociados a la construcción de VIS de tres pisos o menos. En el estudio se analizan tres de los sistemas estructurales más utilizados para la construcción de VIS en Latinoamérica, tales como el sistema tradicional de muros de mampostería confinada, muros de concreto con refuerzo convencional (mallas electrosoldadas) y muros de concreto con fibras de acero. El análisis de presupuestos se realizó con base en las cantidades de obra, los precios unitarios y los ítems particulares de cada sistema estructural. En el estudio también se discuten otros aspectos relevantes de los sistemas estructurales estudiados, tales como el desempeño sísmico, la sostenibilidad, la industrialización, los tiempos de construcción, y la calidad de acabados de los muros.

\section{Características y especificaciones para VIS}

En Colombia, la VIS debe cumplir con los parámetros de calidad especificados en el folleto informativo Núm. 21 de la Oficina del Alto Comisionado para los Derechos Humanos de la ONU (OACDH, 2009). Adicionalmente, el diseño y la construcción de VIS deben incluir el uso sostenible de los recursos naturales. En la tabla 1 se muestran las características que deben cumplir las viviendas de interés social y su normatividad respectiva.

\section{Descripción del prototipo}

Los sistemas estructurales en este estudio corresponden a los sistemas más utilizados para la construcción de VIS en Latinoamérica; es decir, muros de mampostería confinada y muros de concreto reforzados convencionalmente. En la figura 1 se muestra la 
Tabla 1. Características de la VIS

\begin{tabular}{|c|c|c|}
\hline Característica & Descripción & Normatividad \\
\hline $\begin{array}{l}\text { Vivienda segura y } \\
\text { eficiente }\end{array}$ & $\begin{array}{l}\text { Construcción sismorresistente } \\
\text { Uso adecuado del agua } \\
\text { Adecuadas instalaciones eléctricas } \\
\text { Apropiadas instalaciones de iluminación } \\
\text { y alumbrado público }\end{array}$ & $\begin{array}{l}\text { NSR-10 (AIS, 2010) } \\
\text { RAS } 2000 \text { (Minvivienda, 2000) } \\
\text { RETIE (Minminas, 2004) } \\
\text { RETILAP (Minminas, 2009) }\end{array}$ \\
\hline Valor & 135 SMMLV $^{*}$ & $\begin{array}{l}\text { Artículo } 117 \text { de la ley } 1450 \text { de } \\
2011 \text { (Minhacienda y DNP, } \\
\text { 2011) }\end{array}$ \\
\hline Área mínima de lote & $35 \mathrm{~m}^{2}$ & \multirow{2}{*}{$\begin{array}{l}\text { Decreto } 2060 \text { de } 2004 \\
\text { (Minvivienda, 2004) }\end{array}$} \\
\hline Frente mínimo & $3.5 \mathrm{~m}$ & \\
\hline Altura mínima & $\begin{array}{l}2.5 \mathrm{~m} \text { para clima frío y templado } \\
2.7 \mathrm{~m} \text { para clima cálido seco y húmedo }\end{array}$ & \multirow{3}{*}{$\begin{array}{l}\text { Guías de asistencia técnica } \\
\text { para vivienda de interés social } \\
\text { (Minvivienda, 2011) }\end{array}$} \\
\hline Áreas mínimas & $\begin{array}{l}\text { Dormitorio }=7.3 \mathrm{~m}^{2} \\
\text { Cocina }=3.6 \mathrm{~m}^{2} \\
\text { Baño }=2.8 \mathrm{~m}^{2} \\
\text { Cuarto de ropas }=1.1 \mathrm{~m}^{2} \\
\text { Salón comedor }=15.6 \mathrm{~m}^{2} \\
\text { Área múltiple }=14.6 \mathrm{~m}^{2}\end{array}$ & \\
\hline Lado mínimo & $\begin{array}{l}\text { Dormitorio }=2.7 \mathrm{~m} \\
\text { Cocina }=1.5 \mathrm{~m} \\
\text { Baño }=1.2 \mathrm{~m} \\
\text { Cuarto de ropas }=0.8 \mathrm{~m} \\
\text { Salón comedor }=2.8 \mathrm{~m} \\
\text { Zona múltiple }=2.7 \mathrm{~m}\end{array}$ & \\
\hline
\end{tabular}

SMMLV = Salario Mínimo Mensual Legal Vigente (COL \$ 616.000, para el año 2014)

construcción de VIS mediante estos sistemas estructurales. Con base en las ventajas que ofrece el concreto reforzado con fibras de acero para la industrialización de la vivienda, y en el desempeño sísmico observado de muros de concreto reforzados con fibras de acero (Carrillo et al., 2012), en el estudio también se incluyó el sistema estructural de muros de concreto reforzados con fibras de acero.

El prototipo de VIS de este estudio corresponde a una vivienda de dos pisos, incluida la cubierta. La altura de entrepiso del prototipo es de $2.5 \mathrm{~m}$ y el área total construida es de $70.2 \mathrm{~m}^{2}$. La distribución interna del espacio se muestra en la figura 2. Para la selección del prototipo se tomaron en cuenta los lineamientos presentados en la tabla 1.

a)

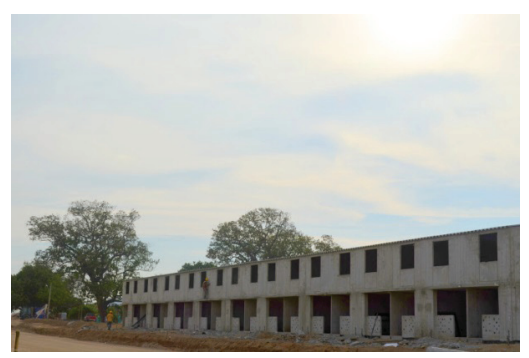

b)

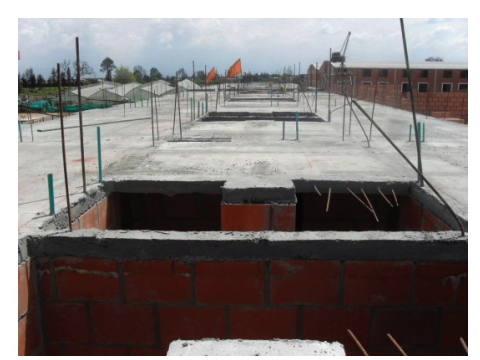

El prototipo se ubicó en una zona de amenaza sísmica intermedia, caracterizada por un coeficiente de aceleración horizontal pico efectiva para diseño, $A_{a^{\prime}}$ de 0.175 . El valor seleccionado de $A_{a}$ corresponde al valor promedio de los coeficientes indicados en NSR-10 para esta zona. Adicionalmente, se supuso que la profundidad del estrato de suelo competente es mayor que $0.70 \mathrm{~m}$.

De acuerdo con Carrillo y Alcocer (2013), estas estructuras tipo caja comúnmente tienen gran resistencia y rigidez lateral y, por tanto, exhiben bajos desplazamientos laterales y demandas de cortante. El bajo nivel de respuesta sísmica ha motivado a los diseñadores a utilizar concretos con resistencia a compresión de 15 a $25 \mathrm{MPa}$, así como muros de $100 \mathrm{~mm}$ de espesor con cuantías de refuerzo en el alma cercanas a la mínima estipulada por la mayoría de los reglamentos de diseño. Por ejemplo, en zonas de amenaza sísmica intermedia y baja, casi la totalidad de los elementos estructurales está controlada por dimensiones y cuantías mínimas de refuerzo.

Figura 1. Construcción de VIS en Colombia, a) muros de concreto (Botero, 2013), b) mampostería confinada (Gómez, 2013) 
Con fines de comparación, para los tres sistemas estructurales se empleó el mismo tipo de cimentación, el cual consiste en un sistema reticular de vigas de concreto reforzado (figura 2a). Cada viga de cimentación sirve como cimiento de un muro o se emplea como viga de cimentación intermedia (VC-C). Con base en la sección E.2.1.5 de NSR-10, esta viga de cimentación intermedia se emplea debido a que el anillo conformado por las vigas VC-3, VC-5, VC-D y VC-B tiene una relación largo/ancho mayor que dos. Según la tabla E.2.2-1 de NSR-10, cada viga tiene sección de $0.30 \times 0.30 \mathrm{~m}$ y está reforzada con cuatro barras de acero corrugado Núm. 4 $\left(f_{y}=420 \mathrm{MPa}\right)$ y estribos Núm. 2 ubicados cada $200 \mathrm{~mm}$ $\left(f_{y}=240 \mathrm{MPa}\right)$. Si se considera que la profundidad del estrato de suelo competente es mayor que $0.70 \mathrm{~m}$, en la base de la viga de cimentación se emplea un relleno de concreto ciclópeo de $0.50 \times 0.50 \mathrm{~m}$ (figura 2a).

En los tres sistemas se decidió usar una placa maciza de contrapiso con espesor de $100 \mathrm{~mm}$ y cuantía mínima $\left(\rho_{\min }\right)$ equivalente a 0.0018 . El área de refuerzo asociado a $\rho_{\text {min }}$ es $180 \mathrm{~mm}^{2} / \mathrm{m}$. Adicionalmente, según la sección C.7.6.5 de NSR-10, la separación del refuerzo principal en losas no debe superar tres veces el espesor de la losa. Por tanto, para el refuerzo de la placa de contrapiso se decidió usar malla tipo M-1.88 ( $\Phi$ 6mm @ $150 \mathrm{~mm}$ en ambas direcciones) y cuya área de refuerzo es $189 \mathrm{~mm}^{2} / \mathrm{m}$.

Para el entrepiso y la cubierta, en los tres sistemas estructurales se decidió usar una placa maciza de concreto reforzado con espesor, $h_{\text {placa }}$ de $150 \mathrm{~mm}$ y $120 \mathrm{~mm}$, respectivamente. Estos valores se obtuvieron de remplazar la luz de diseño, $l$, de $3.38 \mathrm{~m}$ (entrepiso) y $2.88 \mathrm{~m}$ (cubierta) en la ecuación 1. Por facilidad constructiva, en el entrepiso se aproximó a $150 \mathrm{~mm}$. La ecuación 1 fue tomada de la tabla E.5.1-1 de NSR-10 y se supone que la losa está apoyada en una sola dirección con un apoyo continuo.

$h_{\text {placa }}=\frac{l}{24}$

La cuantía de refuerzo de las placas de entrepiso y cubierta se supuso con $\rho_{\min }$ equivalente a 0.0018 , de modo similar a la cuantía utilizada en la mayoría de las losas de estas viviendas. De esta manera, el área de refuerzo asociado a $\rho_{\min }$ de la placa de entrepiso es de $270 \mathrm{~mm}^{2} / \mathrm{m}$, mientras que para la placa de cubierta es de $216 \mathrm{~mm}^{2} / \mathrm{m}$. De acuerdo con la sección C.7.6.5 de NSR-10, la separación del refuerzo principal en losas no debe superar tres veces el espesor del elemento. Por tanto, para el refuerzo superior e inferior de la placa de entrepiso se decidió utilizar malla tipo M-2.95 ( $\Phi 7.5 \mathrm{~mm}$ @ 150mm en ambas direcciones), cuya área de refuerzo es de $295 \mathrm{~mm}^{2} / \mathrm{m}$. Para el refuerzo superior e inferior de la placa de cubierta se utilizó malla tipo M-2.21 ( $\Phi$ 6.5mm @ 150mm en ambas direcciones), cuya área de refuerzo es de $221 \mathrm{~mm}^{2} / \mathrm{m}$.

Finalmente, para los tres métodos constructivos se supuso una escalera con ancho de $0.90 \mathrm{~m}$, espesor de $200 \mathrm{~mm}$, huellas de $280 \mathrm{~mm}$ y contrahuellas de $180 \mathrm{~mm}$. Adicionalmente, se supone que la cuantía mínima de refuerzo, $\rho_{m i n}$ es adecuada, por lo que se utiliza malla M-3.35 ( $\Phi 8.0 \mathrm{~mm} @ 150 \mathrm{~mm}$ en ambas direcciones, área de refuerzo de $336 \mathrm{~mm}^{2} / \mathrm{m}$ ), tanto en la parte superior como en la parte inferior.

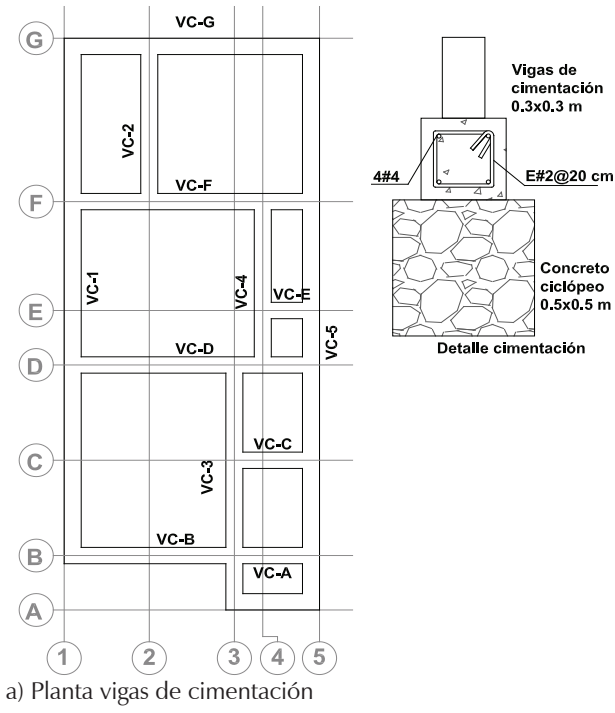

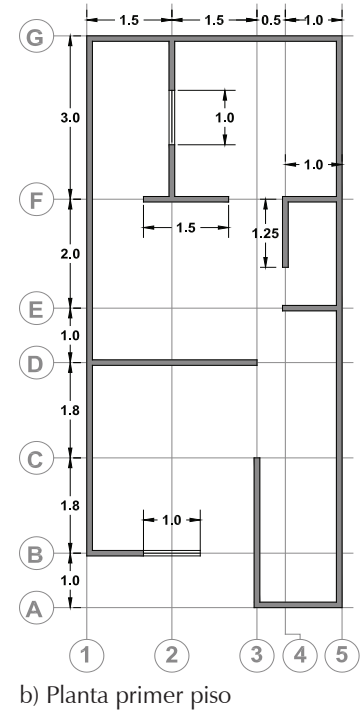

Figura 2. Plantas del prototipo

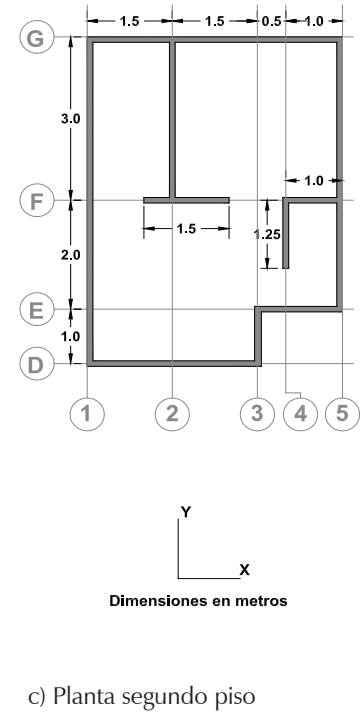




\section{Consideraciones particulares para cada sistema constructivo}

En esta sección se presentan y discuten los resultados del diseño de los muros de concreto para cada uno de los sistemas estructurales estudiados. En el caso de los muros de mampostería confinada, también se presentan los resultados del diseño de los elementos de confinamiento y los elementos no estructurales (parapetos y muros divisorios).

\section{Sistema de mampostería confinada}

\section{Muros de mampostería y elementos de confinamiento}

De acuerdo con lo estipulado en la sección E.3.5 de NSR10, el espesor mínimo de los muros en el primero y segundo piso es de $110 \mathrm{~mm}$ y $95 \mathrm{~mm}$, respectivamente. Con base en las características geométricas de las unidades disponibles en el mercado, en este estudio, el espesor de todos los muros se estableció en $120 \mathrm{~mm}$. En la figuras $3 a$ y $3 \mathrm{~b}$ se muestran los muros escogidos como muros estructurales y los elementos de confinamiento (columnetas y vigas) para el primer y segundo piso.

La longitud mínima de muros, $L_{\text {min }}$ se verifica de acuerdo con la sección E.3.6.4 de NSR-10, y se compara con las longitudes indicadas en los planos. En la tabla 2 se muestran estos valores. De acuerdo con la tabla E.3.61 de NSR-10, el coeficiente $M_{o}$ depende de la zona de amenaza sísmica (intermedia) y del valor de $A_{a}(0.175)$. En la tabla 2 se comprueba que la longitud de los muros de mampostería en cada dirección, $L_{x}$ y $L_{y^{\prime}}$ cumple con este requerimiento.

De acuerdo con los lineamientos indicados en las secciones E.4.3.2 y E.4.4.2 de NSR-10, los elementos de confinamiento (vigas y columnetas) son de concreto reforzado y tienen una sección de $0.12 \times 0.20 \mathrm{~m}$. En la sección E.4.2 de NSR-10 se estipula que el valor mínimo de la resistencia a compresión del concreto, $f_{c^{\prime}}^{\prime}$ y del esfuerzo de fluencia del acero longitudinal y transversal, $f_{y^{\prime}}$ debe ser 17.5 MPa y $240 \mathrm{MPa}$, respectivamente. Para cumplir con los requerimientos mínimos de materiales, el valor de $f_{c^{\prime}}^{\prime}$ y de $f_{y}$ del acero longitudinal y transversal de los elementos de confinamiento es igual a $21 \mathrm{MPa}$, $420 \mathrm{MPa}$ y $240 \mathrm{MPa}$, respectivamente. De acuerdo con la sección E.4.3.4 de la NSR-10, las columnetas se refuerzan con 4 barras Núm. 3 en el sentido longitudinal y estribos Núm. 2 con la siguiente separación: cada 100 $\mathrm{mm}$ en los 6 primeros estribos en las zonas adyacentes a los elementos horizontales, y cada $200 \mathrm{~mm}$ en las otras zonas. De acuerdo con la sección E.4.4.4, las vigas están reforzadas con 4 barras Núm. 3 en el sentido longitudinal y estribos Núm. 2 con la siguiente separación: cada $100 \mathrm{~mm}$ en los primeros $0.5 \mathrm{~m}$ de cada extremo de la viga y cada $200 \mathrm{~mm}$ en el resto de la viga.

\section{Elementos no estructurales}

Los elementos no estructurales corresponden a los parapetos de las dos ventanas del primer piso y al muro divisorio ubicado en el segundo piso. Para conservar la uniformidad en los materiales, estos elementos se planean con el mismo tipo y geometría de la mampostería.
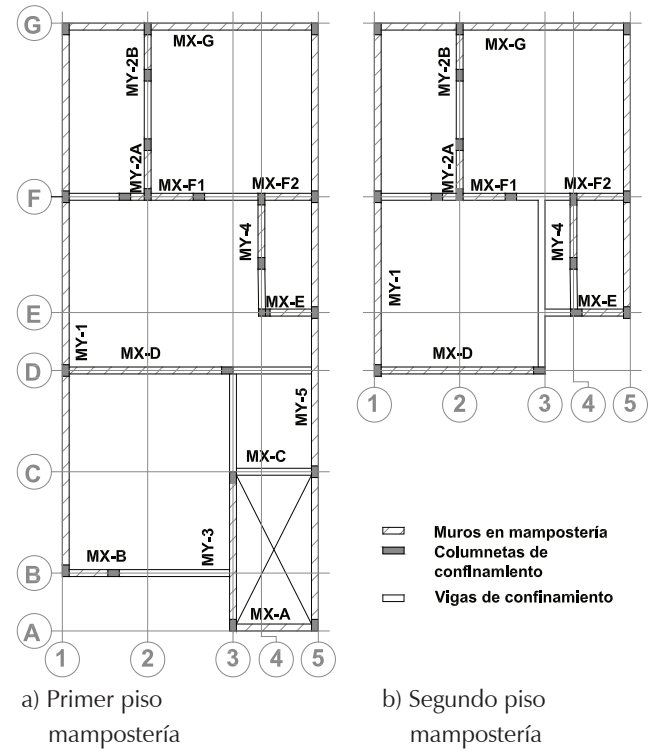

b) Segundo piso mampostería

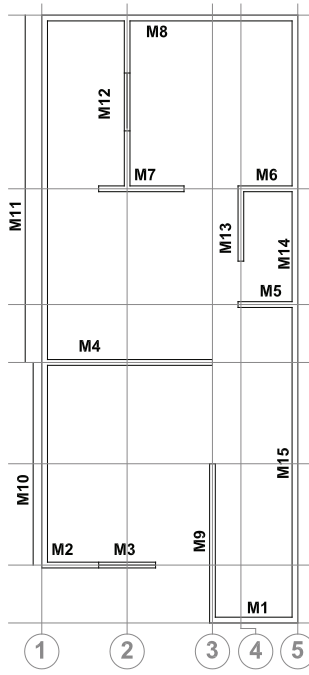

c) Primer piso muros de concreto

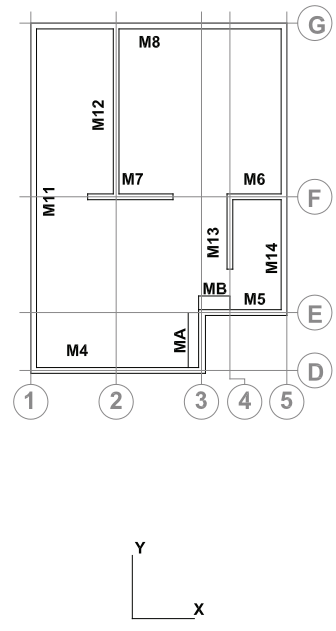

d) Segundo piso muros de concreto
Figura 3. Configuración de los sistemas de mampostería confinada $y$ de muros de concreto 
Tabla 2. Longitud mínima de muros de mampostería

\begin{tabular}{ccccccc}
\hline Piso & Coeficiente Mo & $A_{p^{\prime}} \mathrm{m}^{2}$ & $t, \mathrm{~mm}$ & $L_{\text {min }^{\prime}} \mathrm{m}$ & $L_{x^{\prime}} \mathrm{m}$ & $L_{y^{\prime}} \mathrm{m}$ \\
\hline 1 & 15 & 40.50 & 120 & 5.06 & 13.65 & 26.05 \\
2 & 15 & 25.78 & 120 & 3.22 & 11.67 & 16.40 \\
\hline
\end{tabular}

Adicionalmente, los elementos no estructurales están confinados por columnetas en sus extremos con sección de $0.12 \times 0.20 \mathrm{~m}$. Los parapetos están rematados con vigas o cintas de amarre en la parte superior. De acuerdo con los lineamientos indicados en la sección E.4.5 de NSR-10, las cintas de amarre están reforzadas con 2 barras Núm. 3 en el sentido longitudinal y con estribos Núm. 2 espaciados cada $200 \mathrm{~mm}$.

\section{Sistema de muros de concreto reforzado con ma- lla electrosoldada}

En la figuras 3c y $3 \mathrm{~d}$ se muestra la ubicación de los muros de concreto en la vivienda. Para los sistemas estructurales de muros de concreto reforzados con malla electrosoldada y con fibra de acero, el área mínima de muros en cada dirección se verificó con la ecuación 2, la cual se estipula en la sección 4.15.2 de ACI 314R-11 (2011).

$$
\sum\left(l_{w} \times t_{w}\right) \geq \frac{9 \times V_{i u}}{\sqrt{f_{c}^{\prime}}}
$$

En la ecuación 2:

$$
\begin{aligned}
l_{w} & =\text { longitud del muro } \\
t_{w} & =\text { espesor del muro } \\
V_{i u} & =\text { cortante sísmico horizontal en cada piso }
\end{aligned}
$$

El espesor de los muros, $t_{w}$, es igual a $100 \mathrm{~mm}$ y $V_{u i}$ se calculó mediante el método de la fuerza horizontal equivalente estipulado en el capítulo A.4.3 de NSR-10. Con base en este método, la fuerza sísmica horizontal en cada piso se obtiene de multiplicar el cortante sísmico en la base (masa de la edificación $\times$ aceleración de la gravedad $\times$ el valor espectral de aceleraciones de diseño, $S_{a}$ ) por el coeficiente $C_{v x}$ definido en la sección A.4.3.2 de NSR-10. De esta manera, el valor de $V_{i u}$ corresponde a la suma de las fuerzas laterales desde el piso $i$ hasta la cubierta. En el cálculo de la masa de la edificación se incluyó $25 \%$ de la carga viva para uso residencial, cuyo valor se obtuvo de las tablas B.4.2.1-1 y B.4.2.1-2 de NSR-10. En la tabla 3 se muestran los valores y se comprueba que el área mínima de muros cumple con este requerimiento.

En este caso, todos los muros se diseñan en concreto reforzado con malla electrosoldada y la resistencia a compresión del concreto, $f_{c^{\prime}}$, es igual a $21 \mathrm{MPa}$. Todos los muros cuentan con cuatro tipos de refuerzo: refuerzo a cortante en el alma, refuerzo a cortante por fricción, refuerzo a flexión, y refuerzo por temperatura. Los muros divisorios no estructurales MA, MB y M3, cuentan únicamente con refuerzo a cortante por fricción (sección C.11.6 de NSR-10), y refuerzo por temperatura (sección C.7.12 de NSR-10). Adicionalmente, con base en la disposición e identificación de los muros en las plantas del primer y segundo piso del prototipo (figuras 3 c y $3 d$ ), se hacen las siguientes consideraciones:

a) el muro ubicado sobre el eje 2 entre ejes F y G se analiza como un muro con una abertura,

b) el muro sobre el eje 1 se divide en 2: el muro 10 que llega hasta el eje D con altura de $2.5 \mathrm{~m}$, y el muro 11 que llega hasta el segundo piso con altura de $5 \mathrm{~m}$.

Con propósitos de simplificación y comparación con los demás sistemas estructurales estudiados, los muros se refuerzan a cortante con la cuantía mínima, $\rho_{\text {min }}$ equivalente a $0.20 \%$ en ambas direcciones del muro (vertical y horizontal), la cual se especifica en la sección C.21.9.2.2 de NSR-10 para viviendas de hasta tres pisos. Por tanto, para muros con espesor de $100 \mathrm{~mm}$ se utiliza malla de refuerzo M-2.21, cuya área de refuerzo es de $221 \mathrm{~mm}^{2} / \mathrm{m}^{2}$. De acuerdo con lo estipulado en la sección C.10 de NSR-10, para el refuerzo por flexión se ubican 4 barras Núm. 3 en sentido longitudinal (vertical) y estribos Núm. 2 con la siguiente separación: cada $100 \mathrm{~mm}$ para los 6 primeros estribos en las zonas adyacentes a

Tabla 3. Área mínima de muros de concreto

\begin{tabular}{cccccc}
\hline Piso & $f_{c}^{\prime}, \mathrm{MPa}$ & $V_{i u}, \mathrm{KN}$ & $9 \times V_{i u} / \sqrt{ } f_{c}^{\prime}, \mathrm{mm}^{2}$ & $\sum\left(l_{w w} \times t_{w w}\right)_{x}, \mathrm{~mm}^{2}$ & $\sum\left(l_{w} \times t_{w}\right)_{y}, \mathrm{~mm}^{2}$ \\
\hline 2 & 21 & 198 & $0.000389 \times 10^{6}$ & $1.167 \times 10^{6}$ & $1.640 \times 10^{6}$ \\
1 & 365 & $0.000717 \times 10^{6}$ & $1.365 \times 10^{6}$ & $2.605 \times 10^{6}$ \\
\hline
\end{tabular}


los elementos horizontales, y cada $200 \mathrm{~mm}$ en las otras zonas. Para el refuerzo a cortante por fricción, con base en las solicitaciones características para este tipo de vivienda y en los lineamientos de la sección C.11.6 de NSR-10, se ubica una barra Núm. 3 cada $300 \mathrm{~mm}$ a lo largo de la base de los muros. Para el refuerzo por temperatura se supone que el refuerzo por cortante en el alma es al menos igual al refuerzo por temperatura, puesto que en muros con malla electrosoldada el refuerzo mínimo para retracción por temperatura es de $0.20 \%$.

\section{Sistema de muros de concreto reforzado con fi- bras de acero}

En este caso, todos los muros se diseñan con concreto reforzado con fibras de acero. De modo similar al sistema de muros de concreto con refuerzo convencional, cada muro cuenta con cuatro tipos de refuerzo: refuerzo a cortante en el alma, refuerzo a cortante por fricción, refuerzo a flexión, y refuerzo por temperatura. Para el refuerzo a flexión y el refuerzo de cortante por fricción, se usa la misma configuración de refuerzo utilizada en los muros de concreto con refuerzo convencional.

De acuerdo con la sección C.5.6.6 de NSR-10, es posible utilizar $60 \mathrm{~kg} / \mathrm{m}^{3}$ de fibras de acero para remplazar el refuerzo mínimo por cortante en vigas; sin embargo, no se indican requisitos para muros. Carrillo et al. (2012) han demostrado que el desempeño a cortante de muros de concreto para vivienda de baja altura reforzados con $30 \mathrm{~kg} / \mathrm{m}^{3}$ de fibras de acero es adecuado para las demandas sísmicas intermedias. Por tanto, en este estudio se supone que los muros están reforzados con $30 \mathrm{~kg} / \mathrm{m}^{3}$ de fibras de acero ZP-306 para el refuerzo a cortante. La fibra ZP-306 tiene longitud de $30 \mathrm{~mm}$, diámetro de 0.62 $\mathrm{mm}$, y relación de aspecto (longitud/diámetro) de 48 . Para el refuerzo por temperatura, se supone que el refuerzo por cortante en el alma es al menos igual al refuerzo por temperatura.

\section{Presupuestos de los sistemas estructurales}

Los presupuestos de los tres sistemas estructurales estudiados se elaboraron con información de Construdata (2013); Echeverry et al. (2000); ICH (2013); Meli et al. (2011) y SENA (2003). Los presupuestos incluyeron un cálculo detallado de cantidades con base en los requerimientos estructurales mínimos y los requerimientos constructivos de cada sistema. Adicionalmente, cada presupuesto se elaboró con referencia a los precios, rendimientos (mano de obra) y desperdicios propuestos por Construdata (2013). Sin embargo, la información se ajustó por medio de los valores de herramienta menor.
Los presupuestos se realizaron de acuerdo con los cálculos de cantidades de obra (incluyendo mano de obra), los precios unitarios y los ítems determinados para cada sistema estructural, por lo que se presentan en términos del salario mínimo mensual legal vigente. Los presupuestos se muestran en la tabla 4 .

En el sistema con muros de mampostería confinada, el precio de los ladrillos portantes tipo "LE" con Perforación Vertical se cotizó directamente con un productor reconocido en el mercado. Para los rendimientos y precios de mano de obra y formaleta en los sistemas de muros de concreto reforzado con malla electrosoldada y muros de concreto reforzado con fibras de acero, se utilizaron las recomendaciones de Echeverry et al. (2000). Para determinar el valor del concreto se utilizó una cotización asociada a sistemas industrializados. Esta cotización fue suministrada por una empresa reconocida en el mercado de concreto premezclado. Adicionalmente, para el costo de las fibras de acero se empleó el costo suministrado por un distribuidor autorizado de este tipo de material en Colombia.

\section{Análisis y discusión de resultados}

En esta sección se realiza la comparación y discusión económica y financiera entre los sistemas de mampostería confinada y muros de concreto, así como entre los sistemas de muros de concreto reforzados con malla electrosoldada o con fibras de acero.

\section{Mampostería confinada y muros de concreto} con malla electrosoldada

A partir de los presupuestos de los dos sistemas se observó que el costo de la vivienda prototipo con el sistema de mampostería reforzada fue de 13.5\% (72.5/63.9) mayor que el costo de la vivienda con el sistema de concreto reforzado con malla electrosoldada. Esta variación podría considerarse poco significativa en términos porcentuales; sin embargo, debido a la magnitud de este tipo de proyectos, esta leve variación se amplifica en términos absolutos a medida que la cantidad de unidades de vivienda incrementa. Por tanto, se considera que esta variación podría representar una gran diferencia económica en el momento de planear un mismo proyecto de VIS con alguno de los tres sistemas estructurales estudiados. Por ejemplo, si se construyen 100 casas en mampostería reforzada, el costo total sería 7248 veces el SMMLV; mientras que para 100 casas en concreto reforzado con malla electrosoldada, el costo total sería 6385 veces el SMMLV. Esta diferencia es de aproximadamente 863 SMMLV, equivalentes a aproxi- 
Tabla 4. Análisis de precios unitarios para cada sistema estructural en términos de SMMLV

\begin{tabular}{|c|c|c|c|c|c|c|c|}
\hline Ítem & Descripción & Un & Cant. & $\begin{array}{c}\text { Vr. } \\
\text { unitario }\end{array}$ & $\begin{array}{c}\text { Mamp. confin }{ }^{1} \text {. } \\
\text { Vr. parcial }\end{array}$ & $\begin{array}{l}\text { Muros } \mathrm{CR}^{2} \\
\text { Vr. parcial }\end{array}$ & $\begin{array}{c}\text { Muros CRFA }^{3} \\
\text { Vr. parcial }\end{array}$ \\
\hline 1 & Preliminares & & & & & & \\
\hline 1.1. & Cerramiento malla verde & $\mathrm{m}$ & 5 & 0.029 & 0.147 & 0.147 & 0.147 \\
\hline 1.2 & Descapote & $\mathrm{m}^{2}$ & 45 & 0.037 & 1.680 & 1.680 & 1.680 \\
\hline \multirow[t]{2}{*}{1.3.} & Localización y replanteo & $\mathrm{m}^{2}$ & 45 & 0.103 & 4.638 & 4.638 & 4.638 \\
\hline & & \multicolumn{3}{|c|}{ Subtotal } & 6.465 & 6.465 & 6.465 \\
\hline 2 & Cimentación & & & & & & \\
\hline 2.1. & Excavación zanjas vigas de cimentación & $\mathrm{m}^{3}$ & 18 & 0.040 & 0.723 & 0.723 & 0.723 \\
\hline 2.2 & Vigas en concreto ciclópeo & $\mathrm{m}^{3}$ & 12 & 0.466 & 5.593 & 5.593 & 5.593 \\
\hline 2.3 . & Vigas en concreto reforzado & $\mathrm{m}^{3}$ & 5 & 1.308 & 6.540 & 6.540 & 6.540 \\
\hline 2.4. & Placa contrapiso e $=0.1 \mathrm{~m}$ & $\mathrm{~m}^{2}$ & 45 & 0.080 & 3.602 & 3.602 & 3.602 \\
\hline 2.5 . & $\begin{array}{l}\text { Acero de refuerzo figurado, } \\
420 \mathrm{MPa}\end{array}$ & $\mathrm{kg}$ & 285 & 0.004 & 1.259 & 1.259 & 1.259 \\
\hline \multirow[t]{2}{*}{2.6.} & Malla electrosoldada & $\mathrm{kg}$ & 296 & 0.003 & 1.016 & 1.016 & 1.016 \\
\hline & & \multicolumn{3}{|c|}{ Subtotal } & 18.732 & 18.732 & 18.732 \\
\hline 3 & Mampostería estructural & & & & & & \\
\hline 3.1. & Muro mampostería e $=0.12 \mathrm{~m}$ & $\mathrm{~m}^{2}$ & 129 & 0.073 & 9.353 & & \\
\hline 3.2 . & Elementos confinamiento, columnetas & $\mathrm{m}^{3}$ & 3 & 2.363 & 7.089 & & \\
\hline 3.3. & Elementos de confinamiento (vigas) & $\mathrm{m}^{3}$ & 2 & 1.317 & 2.634 & & \\
\hline \multirow[t]{2}{*}{3.4.} & $\begin{array}{l}\text { Acero de refuerzo figurado, } \\
420 \mathrm{MPa}\end{array}$ & $\mathrm{kg}$ & 637 & 0.004 & 2.814 & & \\
\hline & & \multicolumn{3}{|c|}{ Subtotal } & 21.890 & & \\
\hline 4 & $\begin{array}{l}\text { Elementos no estructurales en } \\
\text { mampostería }\end{array}$ & & & & & & \\
\hline 4.1. & Parapetos & $\mathrm{m}^{2}$ & 2 & 0.030 & 0.059 & & \\
\hline 4.2. & Muro divisorios & $\mathrm{m}^{2}$ & 3 & 0.072 & 0.217 & & \\
\hline \multirow[t]{2}{*}{4.3.} & $\begin{array}{l}\text { Acero de refuerzo figurado, } \\
420 \mathrm{MPa}\end{array}$ & $\mathrm{kg}$ & 5 & 0.004 & 0.022 & & \\
\hline & & \multicolumn{3}{|c|}{ Subtotal } & 0.299 & & \\
\hline 5 & Concreto estructural & & & & & & \\
\hline 5.1. & Muros en concreto e $=0.10 \mathrm{~m}$ & $\mathrm{~m}^{2}$ & 167 & $\begin{array}{r}0.086 \\
0.121^{1}\end{array}$ & & 14.454 & 14.454 \\
\hline 5.2. & Placa entrepiso e $=0.15 \mathrm{~m}$ & $\mathrm{~m}^{2}$ & 41 & $\begin{array}{r}0.126^{2} \\
y^{3}\end{array}$ & 4.954 & 5.094 & 5.094 \\
\hline 5.5. & Placa cubierta e $=0.12 \mathrm{~m}$ & $\mathrm{~m}^{2}$ & 26 & $\begin{array}{r}0.097^{1} \\
0.102^{2} \\
y 3\end{array}$ & 2.513 & 2.634 & 2.634 \\
\hline 5.4 . & $\begin{array}{l}\text { Acero de refuerzo figurado, } \\
420 \mathrm{MPa}\end{array}$ & $\mathrm{kg}$ & $420^{2} 379^{3}$ & 0.004 & & 1.856 & 1.676 \\
\hline 5.5. & Malla electrosoldada & $\mathrm{kg}$ & $\begin{array}{r}594^{1} \\
1142^{2} \\
593^{3}\end{array}$ & 0.003 & 2.039 & 3.919 & 2.036 \\
\hline \multirow[t]{2}{*}{5.6.} & Fibras de acero ZP-306 & $\mathrm{kg}$ & 502 & 0.007 & & & 3.308 \\
\hline & & \multicolumn{3}{|c|}{ Subtotal } & 9.506 & 27.956 & 29.202 \\
\hline 6 & Elementos no estructurales concreto & & & & & & \\
\hline 6.1. & Escalera 1 tramo & $\mathrm{m}^{3}$ & $2^{1}, 1^{2 y 3}$ & $\begin{array}{r}2.030^{1} \\
0.808^{2} \\
y 3\end{array}$ & 4.060 & 0.509 & 0.808 \\
\hline \multirow[t]{2}{*}{6.2.} & Malla electrosoldada & $\mathrm{kg}$ & 66 & 0.003 & 0.227 & 0.226 & 0.227 \\
\hline & & \multicolumn{3}{|c|}{ Subtotal } & 4.286 & 0.735 & 1.035 \\
\hline$\therefore$ & Total costos directos obra & & & & 61.178 & 53.889 & 55.434 \\
\hline$\stackrel{\infty}{\sim}$ & Administración & $10 \%$ & & & 6.118 & 5.389 & 5.543 \\
\hline \multirow{5}{*}{ 导 } & Imprevistos & $5 \%$ & & & 3.059 & 2.694 & 2.772 \\
\hline & Utilidad & $3 \%$ & & & 1.835 & 1.617 & 1.663 \\
\hline & Total AIU & $18 \%$ & & & 11.012 & 9.700 & 9.978 \\
\hline & Valor IVA $16 \%$ sobre utilidad & $16 \%$ & & & 0.294 & 0.259 & 0.266 \\
\hline & Total costos obra & & & & 72.5 & 63.9 & 65.7 \\
\hline
\end{tabular}

${ }^{1}$ Mampostería confinada, ${ }^{2}$ Muros concreto reforzados con malla electrosoldada, $\mathrm{CR},{ }^{3}$ Muros concreto reforzados con

fibras de acero, CRFA 
madamente 14 unidades de vivienda en muros de concreto reforzado con malla electrosoldada.

Cuando se comparan los costos de los muros de mampostería y los muros de concreto, se observa que el costo del concreto para los muros es mayor que el costo del concreto convencional de columnas y vigas de confinamiento. Sin embargo, el costo de la vivienda prototipo es aún menor en los sistemas estructurales basados en muros de concreto reforzado.

Cuando se tiene en cuenta la mano de obra en los sistemas industrializados, los obreros y trabajadores realizan tareas específicas y cíclicas debido a lo repetitivo de las tareas generadas, lo cual genera que cada persona se especialice en un trabajo particular. De esta manera, en el sistema industrializado se sigue un esquema de producción con puestos de trabajo definidos y especializados (figuras $4 \mathrm{a}$ y $4 \mathrm{~b}$ ). Este esquema de producción genera mayor eficiencia, lo que se traduce en reducción de tiempos de trabajo, reducción en desperdicios y tiempos muertos y, al mismo tiempo, reducción en costos.

En este estudio, el costo de formaleta por $\mathrm{m}^{2}$ está asociado a una capacidad de 1000 usos, que es el uso recomendado para el tipo de formaleta empleado. No obstante, el valor de la formaleta se puede reducir considerablemente si se tiene en cuenta que, en el ámbito constructivo, el uso de la formaleta llega a los 1500 usos $\left(360^{\circ}\right.$ en Concreto, 2013). Por tanto, si se considera un número de usos de 1700, el valor de las viviendas con sistema de muros de concreto reforzado con malla electrosoldada o con fibras de acero disminuye a 63.6 y 65.3 veces el SMMLV, respectivamente; es decir, el margen de diferencia de costos entre los muros de concreto reforzados con malla electrosoldada o con fibras de acero y la mampostería se amplía a $14.0 \%$ (72.5/63.6) y $11.0 \%$ (72.5/65.3), respectivamente.

En este estudio, los acabados no se tuvieron en cuenta en los presupuestos realizados. Sin embargo, debido al acabado liso generado por el sistema de formaletas empleado en los muros estructurales de concreto, los pañetes y pintura requeridos para dar acabado a los muros se reducen de manera significativa.

Es importante considerar que los sistemas de muros de concreto usualmente exhiben mejor desempeño sísmico y, por tanto, se caracterizan por tener mayores niveles de seguridad. Por ejemplo, Carrillo y Alcocer (2013) demostraron que es posible proporcionar a la vivienda de concreto las características sismo-resistentes necesarias de acuerdo con el tipo de materiales empleados, la geometría de la estructura y la demanda sísmica sobre los muros. Sin embargo, se debe tener en cuenta que todas las técnicas de construcción deben ser integrales, es decir, tanto el desempeño estructural como sustentable de la vivienda debe ser satisfactorio. Por ejemplo, los sistemas industrializados de muros de concreto están más asociados con desarrollo sostenible, ya que se adapta fácilmente a diferentes ambientes, se promueve la reducción de los costos de operación y mantenimiento, y se generan menores desechos y desperdicios (Carrillo et al., 2013; Carrillo y Alcocer, 2012), lo cual hace que la huella ambiental generada por la actividad de construcción sea menor que la observada al momento de utilizar otros sistemas estructurales. El sistema de mampostería confinada presenta mayor desperdicio de material, debido a las características artesanales propias del sistema constructivo. De esta manera, el valor presupuestado se incrementa considerablemente en comparación con los otros dos sistemas estructurales de muros de concreto.

Adicionalmente, si se tiene en cuenta el mejor comportamiento acústico y la reducción de costos en sistemas de calefacción dentro de las viviendas, se observa que el uso de sistemas estructurales con muros de concreto reforzado presentan ventajas significativas. Por otro lado, la velocidad de construcción también se debe considerar; por ejemplo, en el proyecto Ciudad Equi- a)

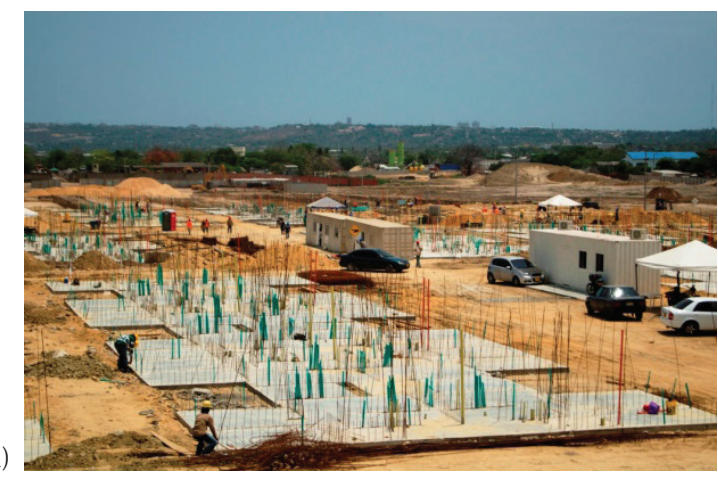

b)

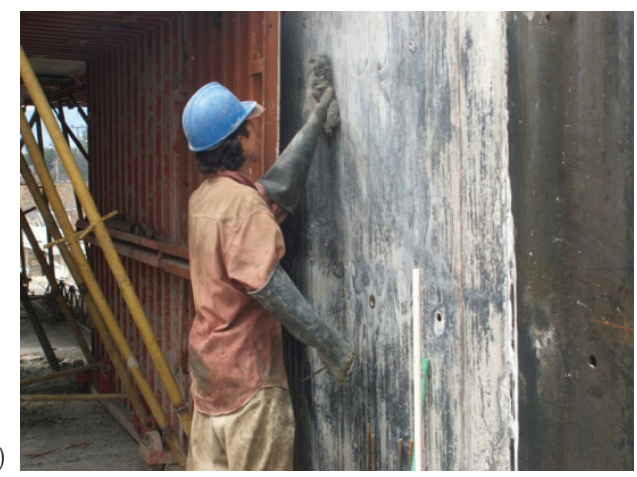

Figura 4. Construcción con muros de concreto en Colombia, a) cimentación (Osorio, 2013), b) muros de concreto $\left(360^{\circ}\right.$ en Concreto, 2013) 
dad se alcanzó un rendimiento de construcción de 42 casas diarias (Altahona, 2013).

Por último, al contrario del sistema de mampostería confinada, Botero (2013) demuestra que el sistema de muros en concreto ofrece la posibilidad de programar efectivamente el costo a un alto grado de detalle, de tal manera que se conoce el día y hora en que se generará cualquier gasto. Si se considera que se tienen pocos materiales para controlar y que sus cantidades son de fácil verificación, dado que los consumos son prácticamente cíclicos y repetitivos, el sistema industrializado de muros de concreto permite ajustar un flujo de caja real, manejar pagos a proveedores eficazmente, requisiciones de materiales a tiempo y un control de presupuesto e inventarios casi en tiempo real, puesto que diariamente se conoce el consumo de un material y, por tanto, no habrá que esperar mucho tiempo para emitir alertas cuando se está fuera del presupuesto.

En general, la vivienda con sistemas industrializados de muros en concreto reforzado es segura ante eventos sísmicos, incentiva la conservación del medio ambiente a partir de una obra más limpia y con menores desperdicios, genera una construcción más rápida, y promueve la reducción de los costos de construcción, operación y mantenimiento. Todas estas ventajas promueven que el costo del sistema industrializado de muros de concreto sea más favorable en comparación con sistemas tradicionales.

\section{Muros de concreto con malla electrosoldada o con fibras de acero}

Como se muestra en la tabla 4, el valor total de los costos de obra obtenido en los dos sistemas estructurales es similar. Sin embargo, el valor para los muros con fibras es $2.9 \%$ (65.7/63.9) mayor. Este incremento se debe principalmente al costo de la fibra de acero. No obstante, la posibilidad de remplazar el refuerzo convencional (barras y mallas) por fibras de acero en otros elementos estructurales tales como vigas, podría reducir el valor de la vivienda, de tal manera que el sistema de muros con fibras resulte más económico. Otro factor importante que se debe considerar en la comparación de los muros de concreto con fibras de acero, es la limpieza en obra y la reducción de desperdicios. Estos aspectos podrían justificar este pequeño sobrecosto de los muros con fibras de acero.

\section{Conclusiones}

Con base en los presupuestos y los análisis realizados en este estudio, se determinó que, desde el punto de vista económico, los sistemas de muros de concreto reforzados con fibras de acero o con malla electrosoldada son más favorables que los sistemas de mampostería confinada. De igual manera, si se tiene en cuenta la necesidad de limpieza en obra, reducción de desperdicios, seguridad, calidad y, principalmente, la necesidad de la industrialización del proceso de construcción de VIS, el sistema de muros de concreto reforzado con fibras de acero presenta menor costo y un panorama más atractivo para los constructores que invierten en este tipo de proyectos. Adicionalmente, el sistema de muros de concreto reforzado con fibras de acero permite obtener no solo beneficios de sostenibilidad y limpieza cuando se compara con los otros sistemas estructurales, sino también beneficios económicos, ya que el costo del sistema es considerablemente inferior al sistema de muros de mampostería confinada. Es importante mencionar que en este estudio se utilizaron dimensiones y cuantías mínimas de refuerzo en los elementos estructurales de las viviendas, tal como se ha demostrado en investigaciones experimentales previas.

\section{Agradecimientos}

Los autores agradecen a la Vicerrectoría de Investigaciones de la Universidad Militar Nueva Granada, UMNG, por el apoyo económico del proyecto IMPING-1574, y al Ing. Cristián Felipe Rodríguez por el apoyo en los cálculos de los presupuestos. El contenido de este artículo representa exclusivamente la opinión de los autores y no refleja la opinión de los patrocinadores y colaboradores.

\section{Referencias}

ACI-Comité 314. Guide to simplified design for reinforced concrete buildings, ACI 314R-11, Farmington Hills, MI, EUA, 2011.

AIS. Reglamento colombiano de construcción sismo resistente, NSR-10, Bogotá, Asociación Colombiana de Ingeniería Sísmica, 2010, $2130 \mathrm{p}$.

Altahona G. Vivienda social en concreto: Ciudad Equidad, Santa Marta, concreto-360 grados-Blog en concreto, Colombia, 2013.

Blog $360^{\circ}$ en Concreto. Formaletas para la construcción con sistemas industrializados, concreto-360 grados-Blog en concreto, Colombia, 2013.

Botero I. Sistema de muros de concreto en la construcción de vivienda, concreto-360 grados-Blog en concreto, Colombia, 2013.

Carrillo J. y Alcocer S. Shear strength of reinforced concrete walls for seismic design of low-rise housing, ACI Structural Journal, volumen 110 (número 3), 2013: 415-425.

Carrillo J., Alcocer S., Aperador W. Propiedades mecánicas del concreto para viviendas de bajo costo. Revista Ingeniería, In- 
vestigación y Tecnología, volumen 14 (número 2), 2013: 285-298.

Carrillo J. y Alcocer S. Revisión de criterios de sostenibilidad en muros de concreto para viviendas sismorresistentes. Revista Ingeniería, Investigación y Tecnología, volumen 13 (número 4), 2012: 479-487.

Carrillo J., Alcocer S., Pincheira J. Shaking table tests of steel fiber reinforced concrete walls for housing, 15th World Conference on Earthquake Engineering, 15WCEE, Lisboa, Portugal, 2012.

Construdata. Índices de costos. Construdata, volumen 165, 2013: 57-69.

DANE. Indicadores económicos alrededor de la construcción, Departamento Administrativo Nacional de Estadística, Colombia. Boletín de prensa, diciembre de 2013.

Echeverry D., Sarria A., Alarcón L., Torres W., Prieto J., Flórez J. Vivienda de interés social: Inventario de sistemas constructivos, Departamento de Ingeniería Civil y Ambiental y Universidad de los Andes, MetroVivienda, Bogotá, 2000.

Gómez J. Placas alveolares: estructuras en concreto pretensado o preesforzado, Concreto-360 grados -Blog en concreto, Colombia, 2013.

ICH. Manual de detallamiento, casas uno y dos niveles de hormigón armado, Instituto del cemento y del hormigón de Chile, Santiago, 2013.

Meli R., Brzev S., Astroza M., Boen T. Crisafulli F, Dai J., Farsi M., Hart T, Mebarki A., Moghadam A.S., Quiun D., Tomazevic M., Yamin L. Seismic design guide for low rise confined masonry buildings, Earthquake Engineering Research Institute, Oakland, California, 2011.

Minhacienda y DNP. Ley 1450 de 2011, Ministerio de Hacienda y Crédito Público y Departamento Nacional de Planeación, Colombia, Diario Oficial Núm. 48102, 2011.
Minminas. Reglamento técnico de iluminación y alumbrado público, RETILAP, Ministerio de Minas y Energía, Colombia, Resolución 181331, 2009.

Minminas. Reglamento técnico de instalaciones eléctricas, RETIE, Ministerio de Minas y Energía, Colombia, Resolución Núm. 18 0398 de 2004 y actualizaciones, 2004.

Minvivienda. Guías de asistencia técnica para vivienda de interés social, Ministerio de Ambiente, Vivienda y Desarrollo Territorial, Colombia, 4 tomos, 2011.

Minvivienda. Decreto 2060 de 2004, Ministerio de Ambiente, Vivienda y Desarrollo Territorial, Colombia, Diario oficial Núm 45590, 2004.

Minvivienda. Reglamento técnico de instalaciones sanitarias, RAS 2000, Ministerio de Ambiente, Vivienda y Desarrollo Territorial, Colombia, Ley 373 de 1997, Decreto 3102 de 1997, Decreto 1052 de 1998, Resolución 1096 de 2000 y actualizaciones, 2000.

Osorio J. Concreto industrializado en la construcción de vivienda, Concreto-360 grados-Blog en concreto, Colombia, 2013.

SPD. Plan de ordenamiento territorial para Santa Fe de Bogotá, D.C. Secretaría de Planeación Distrital, Bogotá D.C. Decreto 619 de 2000, 2000.

SENA. Construcción de casas sismorresistentes de uno y dos pisos. Componentes estructurales que garantizan la sismorresistencia, Servicio Nacional de Aprendizaje, Guía de estudio, Universidad Nacional de Colombia, Medellín, Colombia, 2003.

OACDH Folleto informativo Núm. 21, El derecho a una vivienda digna, Oficina del Alto Comisionado para los Derechos Humanos, Organización de Naciones Unidas (ONU), 2009. 


\section{Este artículo se cita: \\ Citación estilo Chicago}

Carrillo, Julián, Fabián Echeverri, William Aperador. Evaluación de los costos de construcción de sistemas estructurales para viviendas de baja altura y de interés social. Ingeniería Investigación y Tecnología, XVI, 04 (2015): 479-490.

\section{Citación estilo ISO 690}

Carrillo J., Echeverri F., Aperador W. Evaluación de los costos de construcción de sistemas estructurales para viviendas de baja altura y de interés social. Ingeniería Investigación y Tecnología, volumen XVI (número 4), octubre-diciembre 2015: 479-490.

\section{Semblanzas de los autores}

Julián Carrillo. Obtuvo los títulos de ingeniero civil en 2002 por la Universidad Militar Nueva Granada, UMNG, Colombia, el de maestro en ingeniería civil (área de estructuras y sísmica) en 2004 por la Universidad de los Andes, Colombia y el de doctor en ingeniería (área de estructuras) en 2010 por la Universidad Nacional Autónoma de México, UNAM. Actualmente es profesor y director del Grupo de Investigación de Estructuras y Sísmica de la UMNG. Es miembro de tres comités del American Concrete Institute, ACI: 314, Diseño simplificado de edificios; 369, reparación y rehabilitación sísmica; y 374, diseño sísmico basado en desempeño de edificios de concreto.

Fabián Echeverri. Obtuvo el título de ingeniero civil en 2013 por la Universidad Militar Nueva Granada, UMNG, Colombia. Actualmente es joven investigador del Grupo de Investigación de Estructuras y Sísmica de la UMNG y cursa estudios de maestría en ingeniería civil (área de estructuras y sísmica) en la Universidad de Los Andes, Colombia.

William Aperador. Obtuvo los títulos de físico en 2003 y el de maestro en metalurgia y ciencia de los materiales en 2006, por la Universidad Pedagógica y Tecnológica de Colombia en 2006, asimismo el de doctor en ingeniería de los materiales en 2009 por la Universidad del Valle, Colombia. Realizó una pasantía internacional en el centro nacional de investigaciones metalúrgicas (CENIM), en Madrid, España, en el laboratorio de ingeniería de materiales y durabilidad. Actualmente es profesor e investigador del grupo de investigación GMCDP-Volta. Ha sido autor y coautor de más de 80 artículos y ha presentado 40 ponencias internacionales y 38 comunicaciones nacionales. 